\section{Clinical, dermoscopic and histological features \\ of a combined tumor composed \\ of a melanoma in situ, a basal cell carcinoma and an intradermal naevus}

\author{
Massimiliano Scalvenzi, ${ }^{1}$ Claudia Costa, ${ }^{1}$ \\ Franco Palmisano, ${ }^{1}$ Gabriella Fabbrocini, ${ }^{1}$ \\ Mariella Siano, ${ }^{2}$ Stefania Staibano ${ }^{2}$ \\ Departments of ${ }^{1}$ Dermatology and \\ 2Biomorphological and Functional Sciences, \\ Federico II University, Naples, Italy
}

\begin{abstract}
The combined tumors are neoplasms consisting of 2 or more distinct cell population that are intimately admixed. The presence of more than one neoplasm in the same lesion comprising melanocytes and keratinocytes are rare but well documented. We report a case of a 65-year-old man with a melanoma in situ, a basal cell carcinoma and an intradermal naevus in collision that clinically appeared as a single lesion. Clinical, dermoscopic and histological features are discussed.
\end{abstract}

\section{Introduction}

The presence of more than one neoplasm in the same lesion comprising melanocytes and keratinocytes are rare but well documented.

The terminology used for these tumors was confusing until it was recently clarified to contain combined, collision, biphenotypic, and colonized tumors. ${ }^{1}$

Some of these cases may entail more significance than a simple curiosity in the situations in which one of the neoplasm is malignant.

The most common association, naevus and basal cell carcinoma (BCC), is difficult to diagnose clinically.

Also the dermoscopy may not offer important clues to improve the clinical diagnosis.

We present the case of a 65 -year-old man presented a pigmented lesion of the sternal region clinically mimicking a melanoma.

\section{Case Report}

A 65-year-old man was referred to our department for an irregular pigmented lesion on the sternal region. He noted that the lesion was slowly getting larger. He admitted a history of blistering sunburns over the course of his life: in fact his trunk showed a great number of freckles. He had not a personal or a familiar history of melanoma.

It was a $3.5 \times 2.6 \mathrm{~cm}$, asymmetrical lesion with irregular borders. It was barely palpable and composed of brown areas and cutaneous paler regions of regressive origin. Based on the clinical features, a tentative diagnosis of melanoma was made (Figure 1).

The lesion was examined dermoscopically (Figure 2). The dermoscopic evaluation revealed the presence of a polymorphic pattern composed by a pigment network, aymmetrical in its distribuition, globular structures together with regressive white areas; an atypical vascular pattern could be observed within or near the whitish areas; blue-greyish globules occupied the lower aspect of this tumor.

These dermoscopic structures supported the diagnosis of melanoma.

According to these dermoscopic features, the adoption of two algorithms, the three-point and the seven-point checklist, confirmed that the excision was needed. The score of the lesion according to the three-point check list was as follows: the asymmetry of color and structure in one or two perpendicular axes, 1; atypical network with irregular holes and thick lines, 0 ; and blue-white structures, 1 . The total score of our lesion was 2 (cut-off value for suspicious lesion should undergo a biopsy, 2). The seven-check list pointed the probable presence of melanoma: the presence of an atypical vascular pattern (major criterion=2), regression areas (minor criterion=1) and globular structures (minor criterion=1) gives a total score of 4 (cut-off value for suspicious lesion, 3 ).

The lesion was surgically excised. Histopathological examination showed, within the epidermis, focal areas of early basal cell carcinoma, mostly superficial as well as areas of basaloid proliferation (Figures 3B,C,E). Intermixed to these areas, we can also observe an atypical lentiginous melanocytic proliferation with multifocal tendency to intraepithelial growth (Hutchinson's freckle-early in situ melanoma, Figure 3A,C,D,E). Images of a residual dermal naevus were also present in several sections of the lesion (Figure 3F). At immunohistochemical study, the melanocytic lesion showed a strong reactivity for HMB45 in the radial growth phase and, where recognizable, in the vertical growth phase.

A diagnosis of a combined tumor was made: in this lesion we have features of BCC, intradermal naevus and melanoma in situ.

\section{Discussion}

The nomenclature of these neoplasm was previously confusing as many of the terms had been used interchangeably until the terminology was recently defined by Satter et al. ${ }^{1}$
Correspondence: Massimiliano Scalvenzi, Department of Dermatology,

via Federico II University, Naples, 80131, Italy. Tel/Fax: +39.081.7462442.

E-mail: scalvenz@unina.it

Key words: melanoma, superficial basal cell carcinoma, combined tumor.

Conflict of interests: the authors report no potential conflict of interests.

Received for publication: 12 September 2012.

Revision received: 10 November 2012.

Accepted for publication: 12 November 2012.

This work is licensed under a Creative Commons Attribution NonCommercial 3.0 License (CC BYNC 3.0).

(C) Copyright M. Scalvenzi et al., 2012

Licensee PAGEPress, Italy

Dermatology Reports 2012; 4:e11

doi:10.4081/dr.2012.e11

Collision tumors: defined as the merging of 2 originally separate, adjacent, and often well demarcated neoplasms within close proximity of each other. ${ }^{1}$

- Combined tumors: a neoplasm consisting of 2 or more distinct cell population that are intimately admixed. ${ }^{1}$

- Colonized tumors: these tumors consist of 1 tumor cell population permeating and colonizing an underlying second tumor cell population, for instance a melanoma in situ colonizing an underlying BCC. ${ }^{1,2}$

- Biphenotypic tumors: combined tumors consisting of 2 or more population of tumor cells that arise from a common precursor and undergo subsequent divergent differentiation. ${ }^{1}$

Based on these criteria, some of the tumors previously reported in literature, in which the

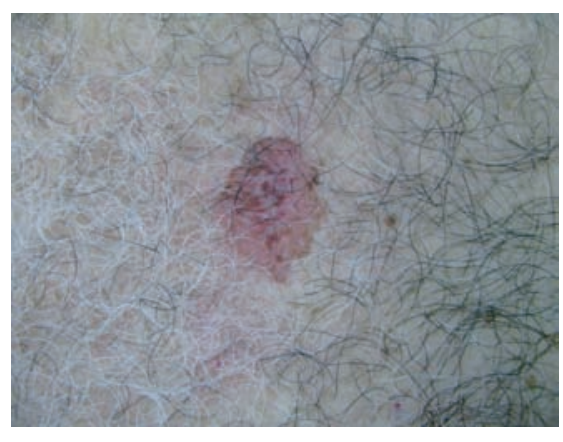

Figure 1. Asymmetrical lesion with irregular borders on the sternal region. It was barely palpable and composed of brown areas and cutaneous paler regions of regressive origin. 


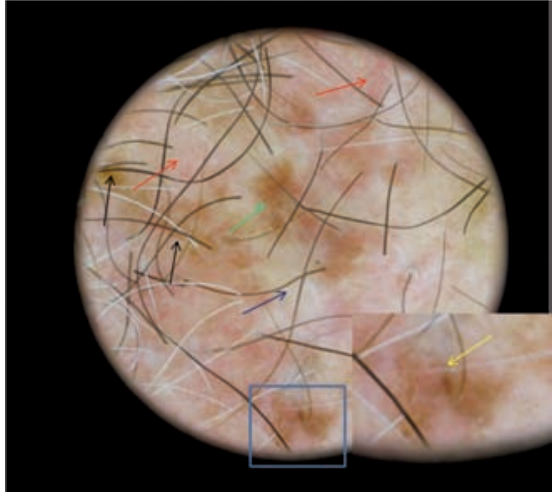

Figure 2. The dermoscopic evaluation revealed the presence of a polymorphic pattern composed by a pigment network, asymmetrical in its distribuition (black arrows), globular structures together with regressive white areas (blue arrow); an atypical vascular pattern (red arrows) could be observed within or near the whitish areas. These dermoscopic structures supported the diagnosis of melanoma. Rare globular structures only in retrospect can be interpreted as the bluegrey ovoid nests sometimes observed in basal cell carcinoma (blue square); also the short and fine telangectasias and shiny white to red areas in the lower portion of the tumour (yellow arrow) can be referred to a basal cell carcinoma while the homogeneous brown pigmentation on the centre of the lesion is attributable to the intradermal naevus (green arrow).

terminology collision and combined was often used with confusion, have been recategorized. ${ }^{3}$

While a combined tumor appears as a single lesion and its 2 or more cell populations can be only histologically identified, a tumor can be already assumed as collisional after the clinical and dermoscopic examination.

Dermoscopy is a noninvasive method that enables clinicians to evaluate morphological features not visible to the clinical evaluation, thus enhancing the diagnosis of nearly all pigmented skin lesions. This technique offers the advantage of differentiating melanocytic from nonmelanocytic lesions, as well as benign from malignant pigmented and non pigmented skin lesions. There are, however, dermoscopic features that can be found in both benign and malignant lesions and in both melanocytic and nonmelanocytic lesions. ${ }^{4}$ In our lesion we describe a melanoma in situ, a BCC and an intradermal naevus in collision that clinically appeared as a single lesion. The dermoscopy did not improve the diagnostic accuracy vs. clinical examination: in fact, we saw only rare globular structures that could only in retrospect be interpreted as the blue-grey ovoid nests sometimes observed in $\mathrm{BCC} ;{ }^{5-7}$ also the short and fine telangectasias and shiny white to red areas in the lower portion of the tumor could be referred
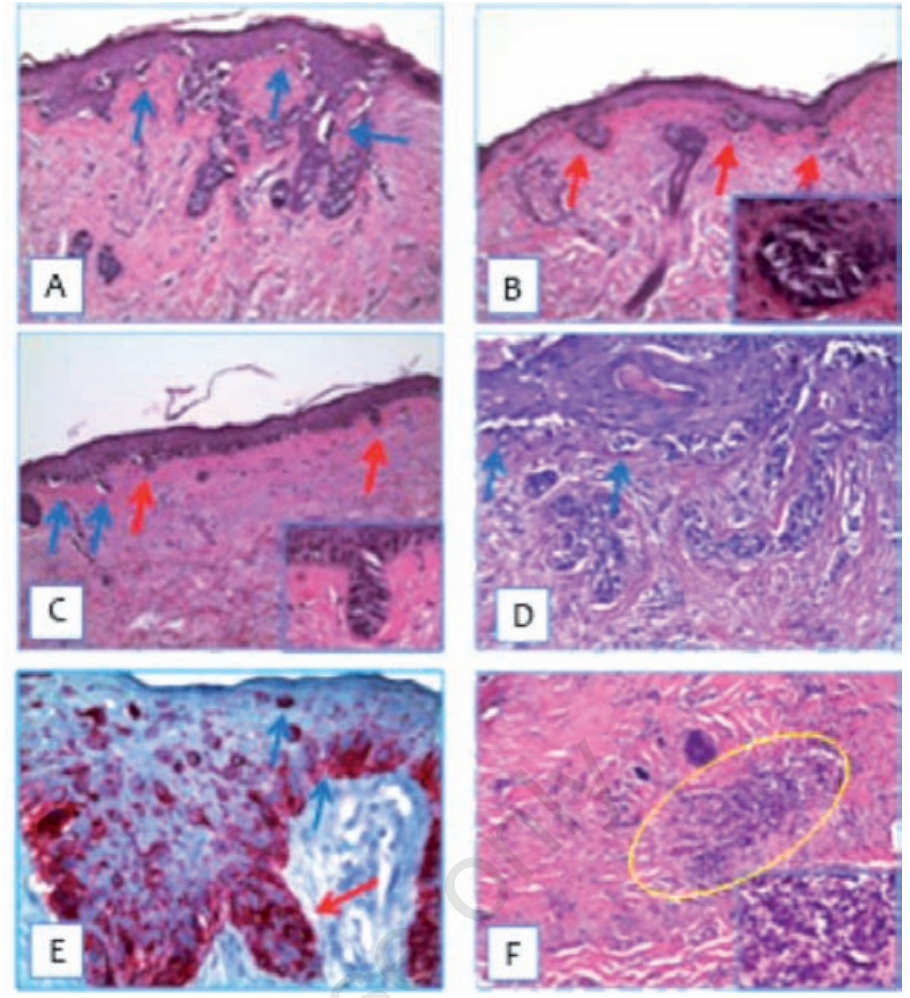

Figure 3. Multiple areas of basaloid proliferation and early superficial basal cell carcinoma (B,C,E; red arrows) are mixed with Hutchinson's lentigo with focal intraepithelial (in situ) melanoma (A,C,D,E; blue arrows), strongly immunoreactive with HMB45. A residual dermal naevus was also detectable in several sections of the same lesion ( $F$, yellow circle).

to a BCC while the homogeneous brown pigmentation on the center of the lesion is attributable to the intradermal naevus. In addition, the atypical vascular pattern, the regression areas and the presence of an irregular pigment network was indicative of a melanoma. Melanoma and BCC are 2 tumors that classically promoted by sunburns. Because sun-damaged changes promote both tumors, their development at the same site on sun-exposed skin, although fortuitous, can easily be explained. ${ }^{8}$ The clinical diagnosis of these cutaneous tumors remains very difficult, and the dermoscopy could not be helpful in these cases. It is difficult to assess the prognosis of these tumors due to their rarity. Some argue these neoplasms should be considered separately. ${ }^{8}$ Given these uncertainties, a follow-up of these lesions after the complete excision is strictly recommended to ascertain the true prognosis.

\section{References}

1. Satter EK, Metcalf J, Lountzis N, et al. Tumors composed of malignant epithelial and melanocytic populations: a case series and review of the literature. J Cutan
Pathol 2009;36:211-9.

2. Burkhalter A, White WL. Malignant melanoma in situ colonizing basal cell carcinoma. Asimulator of invasive melanoma. Am J Dermatopathol 1997;17:303-7.

3. Cornejo KM, Deng AC. Malignant melanoma within squamous cell carcinoma and basal cell carcinoma: is it a combined or collision tumor? A case report and review of the literature. Am J Dermatopathol. 2012 May 14.

4. Argenziano G, Soyer HP, De Giorgi V et al. Dermoscopy: a tutorial. Milan: Edra Medical Publishing \& New Media; 2000.

5. Menzies SW. Dermoscopy of pigmented basal cell carcinoma. Clin Dermatol 2002; 20:268-9.

6. Peris K, Altobelli E, Ferrari A et al. Interobserver agreement on dermoscopic features of pigmented basal cell carcinoma. Dermatol Surg 2002;28:643-5.

7. Scalvenzi M, Lembo S, Francia MG, Balato A. Dermoscopic patterns of superficial basal cell carcinoma. Intern $\mathrm{J}$ Dermatol 2008;47:1015-8.

8. Belisle A, Gautier MS, Farida G et al. A collision tumor involving basal cell carcinoma and lentigo maligna melanoma. Am J Dermatopathol 2005;27:319-21. 